\title{
O6-methylguanine-DNA methyltransferase is downregulated in transformed astrocyte cells: implications for anti-glioma therapies Ken Sasai*1, Tsuyoshi Akagi ${ }^{2}$, Eiko Aoyanagi ${ }^{1}$, Kouichi Tabu ${ }^{1}$, Sadao Kaneko ${ }^{3}$ and Shinya Tanaka*1
}

Address: ${ }^{1}$ Laboratory of Molecular and Cellular Pathology, Hokkaido University Graduate School of Medicine, W15 N7, Kita-ku, Sapporo 0608638, Japan, ${ }^{2}$ KAN Research Institute Inc., 6-7-3 Minatojima-minamimachi, Chuo-ku, Kobe 650-0047, Japan and ${ }^{3}$ Kashiwaba Neurosurgical Hospital, 15-7-20, Tsukisamu E1, Toyohira-ku, Sapporo 062-8513, Japan

Email: Ken Sasai* - ksasai@med.hokudai.ac.jp; Tsuyoshi Akagi - t-akagi@kan.eisai.co.jp; Eiko Aoyanagi - eiko-a@patho2.med.hokudai.ac.jp; Kouichi Tabu - k-tabu@med.hokudai.ac.jp; Sadao Kaneko - srkaneko@seagreen.ocn.ne.jp; Shinya Tanaka* - tanaka@med.hokudai.ac.jp

* Corresponding authors

Published: 5 June 2007

Molecular Cancer 2007, 6:36 doi:10.1 186/1476-4598-6-36
Received: 26 April 2007

Accepted: 5 June 2007

This article is available from: http://www.molecular-cancer.com/content/6/1/36

(C) 2007 Sasai et al; licensee BioMed Central Ltd.

This is an Open Access article distributed under the terms of the Creative Commons Attribution License (http://creativecommons.org/licenses/by/2.0), which permits unrestricted use, distribution, and reproduction in any medium, provided the original work is properly cited.

\begin{abstract}
Background: A novel alkylating agent, temozolomide, has proven efficacious in the treatment of malignant gliomas. However, expression of $0^{6}$-methylguanine-DNA methyltransferase (MGMT) renders glioma cells resistant to the treatment, indicating that identification of mechanisms underlying the gene regulation of $M G M T$ is highly required. Although glioma-derived cell lines have been widely employed to understand such mechanisms, those models harbor numerous unidentified genetic lesions specific for individual cell lines, which complicates the study of specific molecules and pathways.

Results: We established glioma models by transforming normal human astrocyte cells via retroviral-mediated gene transfer of defined genetic elements and found that MGMT was downregulated in the transformed cells. Interestingly, inhibitors of DNA methylation and histone deacetylation failed to increase MGMT protein levels in the transformed astrocyte cells as well as cultured glioblastoma cell lines, whereas the treatment partially restored mRNA levels. These observations suggest that downregulation of MGMT may depend largely on cellular factors other than promoter-hypermethylation of MGMT genes, which is being used in the clinic to nominate patients for temozolomide treatment. Furthermore, we discovered that Valproic acid, one of histone deacetylase inhibitors, suppressed growth of the transformed astrocyte cells without increasing MGMT protein, suggesting that such epigenetic compounds may be used to some types of gliomas in combination with alkylating agents.
\end{abstract}

Conclusion: Normal human astrocyte cells allow us to generate experimental models of human gliomas by direct manipulation with defined genetic elements, in contrast to tumor-derived cell lines which harbor numerous unknown genetic abnormalities. Thus, we propose that the study using the transformed astrocyte cells would be useful for identifying the mechanisms underlying MGMT regulation in tumor and for the development of rational drug combination in glioma therapies. 


\section{Background}

Gliomas, accounting for $30 \%$ of adult primary brain tumors, are the most common primary tumors of the central nervous system and are classified into four clinical grades, with the most aggressive and lethal tumors being grade IV glioblastoma multiforme (GBM) $[1,2]$. The median survival of GBM patients is less than one year from initial diagnosis, and many of the commonly used chemotherapeutic agents have limited effects on these malignant tumors [3]. Recently, there has been increasing hope that temozolomide, a novel alkylating agent, will prove efficacious in the treatment of human glioma $[4,5]$. However, a number of studies have suggested that, in tumors, $\mathrm{O}^{6}$-methylguanine-DNA methyltransferase (MGMT) provides resistance to treatment with temozolomide, unless expression is lost by promoter methylation or there is direct inhibition of MGMT activity [6]. Considering the attractive efficacy of temozolomide, one of the greatest challenges facing the field may be to identify therapeutic agents that suppress MGMT expression, as such drugs may sensitize resistant glioma cells to temozolomide. Thus, establishment of more sophisticated systems to understand the functions and regulation of MGMT are highly desired.

Normal human cells, genetically modified by retroviralmediated gene transfer, have proven important, because such systems are useful for identifying factors directly contributing to tumorigenesis, in contrast to tumor-derived cell lines which harbor numerous unknown genetic abnormalities $[7,8]$. GBMs are believed to arise from astrocyte cells by means of stepwise accumulation of genetic abnormalities [4,9], and immortalized normal human astrocyte (NHA) cells had been established by introducing the telomerase catalytic subunit (hTERT) in combination with human papillomavirus E6/E7 to inactivate both $\mathrm{p} 53$ and $\mathrm{pRb}$ pathways [10]. It had been systematically demonstrated that the immortalized NHA cells, expressing activated Ras (H-RasV12) or expressing both $\mathrm{H}$-RasV12 and an active form of Akt (myrAKT), formed tumors consistent with human anaplastic astrocytoma or GBM in intracranial- and flank-xenografts models $[10,11]$. These studies indicate that such systems are useful for glioma research to understand direct functions and regulation of genetic elements during transformation and gliomagenesis.

Here we created similar experimental models using NHA cells by introducing the simian virus 40 early region (SV4OER) instead of human papillomavirus E6/E7. The SV4OER encodes both small-t antigen, a suppressor of protein phosphatase $2 \mathrm{~A}$ (which is downregulated in half of human glioma) [12] and large-T antigen, which directly binds to and inactivates p53, as well as pRB and the closely related proteins p107 and p130 [13]. Using such genetically modified NHA cells, we demonstrate that MGMT is downregulated during oncogene-mediated transformation of astrocyte cells. Since our results indicate that downregulation of MGMT expression was not primarily dependent upon promoter hypermethylation, inhibitors of DNA methylation or histone deacetylases (HDACs) may be used in combination with alkylating agents for improved treatment of some GBM cases. We propose that the NHA cell systems are useful for investigating the mechanisms underlying MGMT expression and for improving glioma therapies.

\section{Results}

To create an experimental model of human gliomas, NHA cells were introduced with genes for hTERT (T), SV4OER (S), H-RasV12 (R), and myrAKT (A) (Figure 1A; NHA/TS, NHA/TSR, NHA/TSRA cells). The NHA/TS cells grew much faster than parental NHA cells, exhibiting continuous growth beyond population-doubling 50 (data not shown). However, the NHA/TS cells did not form colonies in soft agar or tumors in xenografts into recipient nude mice (Figure 1B; Table 1), indicating that the NHA/ TS cells were immortalized but not transformed. In contrast, the NHA/TSR and NHA/TSRA cells formed a considerable number of colonies in soft-agar, as well as tumors in flank-xenografts into nude mice (Table 1). NHA/TSRA cells displayed much more refractile morphology and loose attachment to the culture dish relative to NHA/TSR cells (Figure 1B). Although the difference in the anchorage-independent growth property was quite subtle between NHA/TSR and NHA/TSRA cells (Table 1), the histopathological features of two types of flank xenografts were clearly different. In tumor tissues formed by NHA/

Table I: Summary of soft agar colony formation assay and xenograft propagation experiment: NHA and NIH3T3 cells infected with retroviral vectors expressing hTERT (T), SV40ER (S), H-RasVI 2 (R), myrAKT (A), and/or MGMT as well as parental NHA cells were subjected to the soft-agar colony formation and xenograft propagation assays.

\begin{tabular}{lcc}
\hline Cell types & Colony numbers $^{a}$ & \% Tumor incidence $(n)^{b}$ \\
\hline NHA (parental) & 0 & ND \\
NHA/TS & 0 & $0(3)$ \\
NHA/TSR & $664 \pm 19$ & $100(3)$ \\
NHA/TSRA & $736 \pm 51$ & $100(3)$ \\
NHA/TSR + MGMT & $649 \pm 34$ & ND \\
NIH3T3/R & $1440 \pm 56$ & ND \\
NIH3T3/R + MGMT & $1344 \pm 88$ & ND \\
\hline
\end{tabular}

aNHA $\left(2 \times 10^{4}\right)$ and NIH3T3 cells $\left(1 \times 10^{4}\right)$ were plated in soft-agar ( $0.36 \%$ top agar containing $5 \%$ fetal calf serum in $60-\mathrm{mm}$ dishes) and incubated for 21 days and 14 days, respectively. Colonies were stained with 3-(4,5-dimethylthiazol-2-yl)-2,5-diphenyltetrazolium bromide, and the numbers of stained colonies were counted. Results represent mean \pm SD from two independent triplicate experiments.

b3 $\times 10^{6}$ cells were injected s.c. into nude mice. $n$, number of animals treated. ND, not done. 


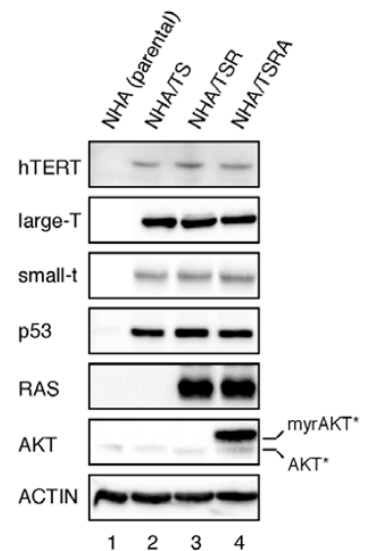

B

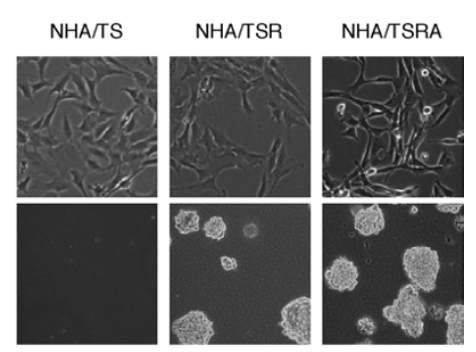

Figure I

Establishment of immortalized and transformed NHA cells. (A) Protein extracts ( $10 \mu \mathrm{g})$ from NHA cells infected with retroviral vectors expressing indicated genes (T, hTERT: S, SV40ER: R, H-RasVI2; A; myrAKT) were analyzed by immunoblotting. Asterisk, active form of AKT (myrAKT) was distinguishable from wild-type form. (B) The morphologies (top panels) and anchorage-independent growth properties (bottom panels) of NHA/TS, NHA/TSR, and NHA/TSRA cells are shown.

TSR cell injection, atypical astrocytic cells with higher cellularity exhibits histopathological features of Grade III glioma as anaplastic astrocytoma of WHO classification. On the other hand, the TSRA-xenografts composed of similar atypical astrocytes displayed numerous areas of necrosis with nuclear pseudo-palisading pattern. Consistent with the histological feature as GBM, the immunohistochemical index of Ki-67 positivity is higher in NHA/TSRA tumors than those of NHA/TSR derived tumors (Figure 2).

Using the NHA cell system described above, we found that the mRNA level of MGMT was decreased in immortalized (NHA/TS) and transformed (NHA/TSR and NHA/TSRA) cells and that MGMT expression was also downregulated in the flank xenografts derived from NHA/TSR and NHA/ TSRA cells (Figure 3A). Immunoblot analyses also demonstrated that introduction of SV40ER decreased MGMT level in NHA cells and that the levels were further decreased in transformed NHA cells (Figure 3B). Conversely, in human lung fibroblasts TIG3/T cells (TIG3 cells expressing hTERT) [14], neither SV40ER nor H-RasV12 suppressed MGMT expression (Figure 3B), suggesting the astrocyte-specific mechanisms of MGMT regulation. This is an interesting observation as TIG3/TS cells are refractory to Ras-induced genetic alteration $[15,16]$, morphological changes [17] and thereby cellular transformation [14]. Although such observation prompted us to further test the tumor suppressive functions of MGMT, the additional
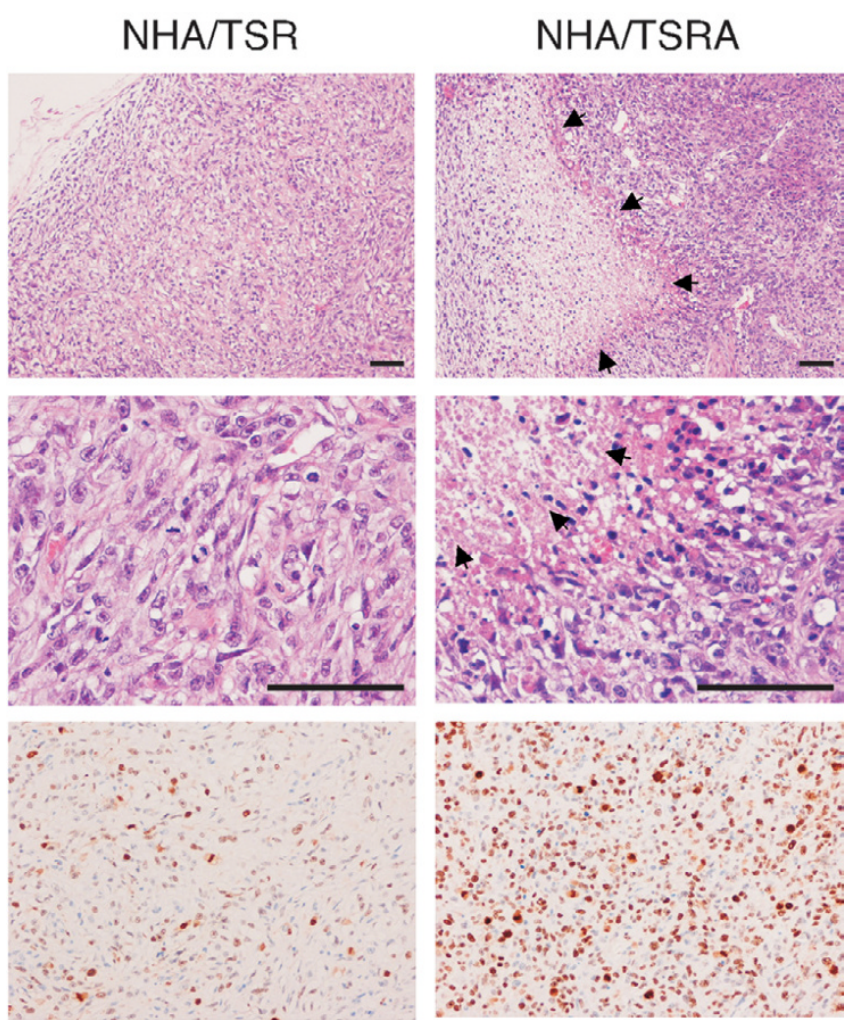

Figure 2

Histological features of s.c. xenografts derived from the transformed NHA cells. Formalin-fixed paraffinembedded tissue sections (5- $\mu \mathrm{m}$-thick) of xenografts derived from NHA/TSR (left) and NHA/TSRA (right) cells were subjected to histological analyses. Low- (top) and high- (middle) magnification images (H\&E staining; scale bar, $200 \mu \mathrm{m}$ ) and immunohistochemistry using an anti-Ki-67 antibody (bottom) are shown.

expression of MGMT in NHA/TSR and NIH3T3/R cells (NIH3T3 cells transformed by H-RasV12) failed to suppress anchorage-independent growth (Table 1), suggesting that MGMT may be unable to restore transformed phenotypes. In fact, tumors have long been noted to be heterogeneous in MGMT expression [5,6], and approximately half of malignant gliomas expressed MGMT [18]. However, it is possible that the mechanisms underlying resistance to downregulation of MGMT may render TIG3 fibroblasts refractory with respect to oncogene-mediated transformation, because MGMT functions to protect normal cells from exogenous carcinogens [6]. In support of this idea is the observation that MGMT mRNA was downregulated in v-fos-transformed $[19,20]$ and Ras-transformed (TA unpublished data) rodent fibroblasts, showing highly malignant phenotypes.

To understand the mechanism underlying the downregulation of MGMT in transformed NHA cells, we first ana- 

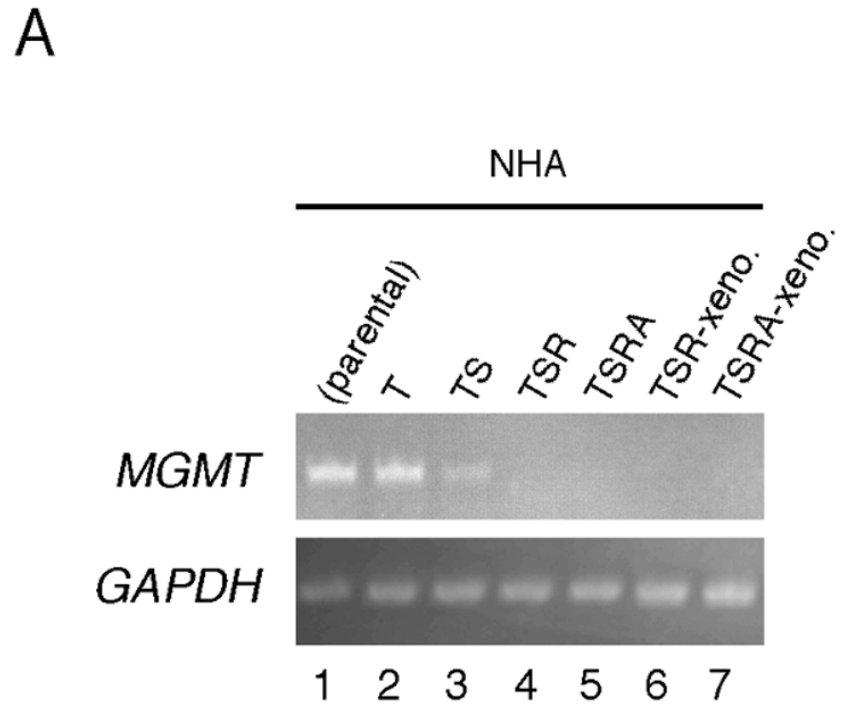

B

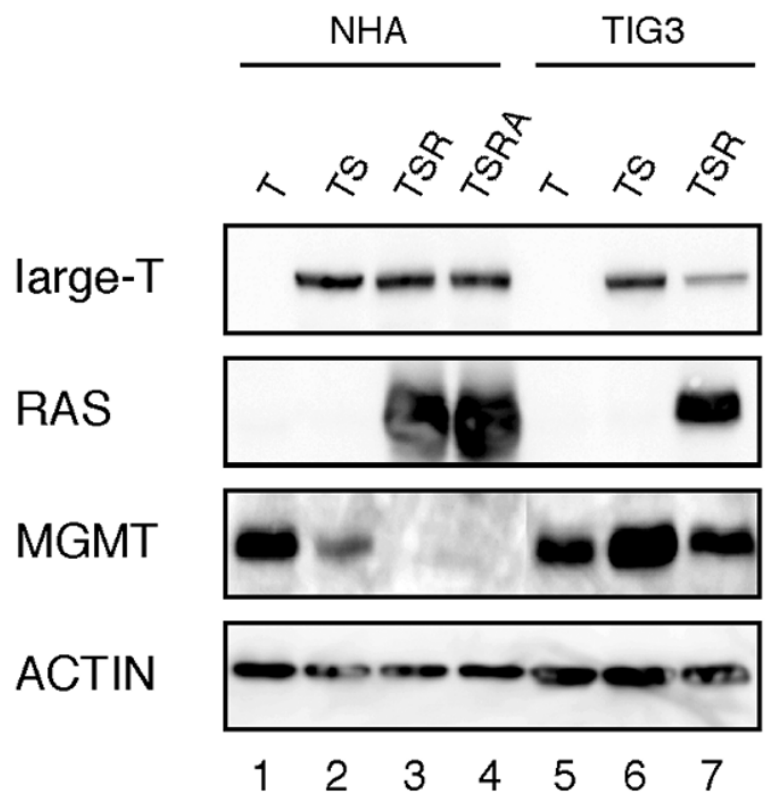

Figure 3

MGMT is downregulated in immortalized and transformed NHA cells. (A) The expression of MGMT mRNA in NHA (parental; lane I), NHA/T (lane 2), NHA/TS (lane 3), NHA/TSR (lane 4), and NHA/TSRA cells (lane 5) were analyzed by semi-quantitative RT-PCR. The levels in flank xenografts derived from the NHA/TSR and NHA/TSRA cells were also tested (lanes 6 and 7). (B) Protein extracts ( $10 \mu \mathrm{g})$ from NHA (lanes I-4) and TIG3 cells (lanes 5-7) infected with retroviral vectors expressing indicated genes ( $T$, hTERT: S, SV40ER: R, H-RasVI2; A; myrAKT) were analyzed by immunoblotting. lyzed the MGMT expression following treatment with 5aza-2'-deoxycytosine (5-aza-dC) and/or Valproic acid (VPA), because it has been widely acknowledged that aberrant methylation of MGMT-promoter contributes to the gene expression changes [6]. Inhibitors of HDACs, such as VPA and trichostatin A, act synergistically with 5aza-dC (a DNA methyltransferase inhibitor and demethylating agent in dividing cells) to further increase the expression of genes silenced in association with promoter hypermethylation [21]. Reverse transcriptase (RT)-PCR analysis revealed that treatments with 5 -aza-dC alone or combined treatment with 5-aza-dC/VPA slightly increased MGMT mRNA levels, when the PCR was performed in a condition where the positive control reaction (mRNA from the NHA/TS cells) was saturated (data not shown), suggesting that the downregulation may have been partially mediated by promoter hypermethylation. However, combined treatment with 5-aza-dC/VPA was insufficient to restore MGMT protein levels in NHA/TSR and NHA/ TSRA cells whereas the treatment clearly induced acetyltation and demethylation of histone H3 (Figure 4A), suggesting that additional factors, which are expressed in the parental NHA cells but inactivated in the transformed NHA cells, may be required to increase MGMT protein levels.

In an attempt to identify such missing factors contributing to gene regulation of MGMT, we tested if the expression of wild type p53 restores MGMT levels, as MGMT was slightly decreased in the NHA/TS cells where p53 is inactivated by large $\mathrm{T}$ antigen (Figure 3 ). Although several glioma studies have demonstrated correlations between inactivation of the p53 pathway and lower levels and/or activity of MGMT [22-24], the introduction of wild type p53 failed to restore MGMT protein levels in transformed NHA cells (Figure 4B). We also confirmed previous reports that both U87MG (harboring wild type p53) and U251MG (harboring p53 mutations) glioma cells expressed low or no levels of MGMT [25-27]. We further found that treatment with 5-aza-dC/VPA failed to induce MGMT protein expression in both cell lines (Figure 4C), whereas the treatment slightly increased the mRNA levels (by RT-PCR; data not shown). Since gliomas harboring p53 mutations and immuno-positive for p53 (G2, G7, and G8) as well as gliomas harboring wild type p53 and immuno-negative for p53 (G4 and G10) expressed MGMT mRNA (Figure 5, Table 2), MGMT might be regulated primarily by the factors other than p 53 in gliomas. Conversely, MGMT is clearly downregulated in normal astrocyte cultures from p53 deficient mice [28], suggesting the differences in the regulation of MGMT between normal and transformed cells.

Since MGMT seems to be regulated in several ways, it is possible that inhibitors of HDACs may be used for thera- 
A

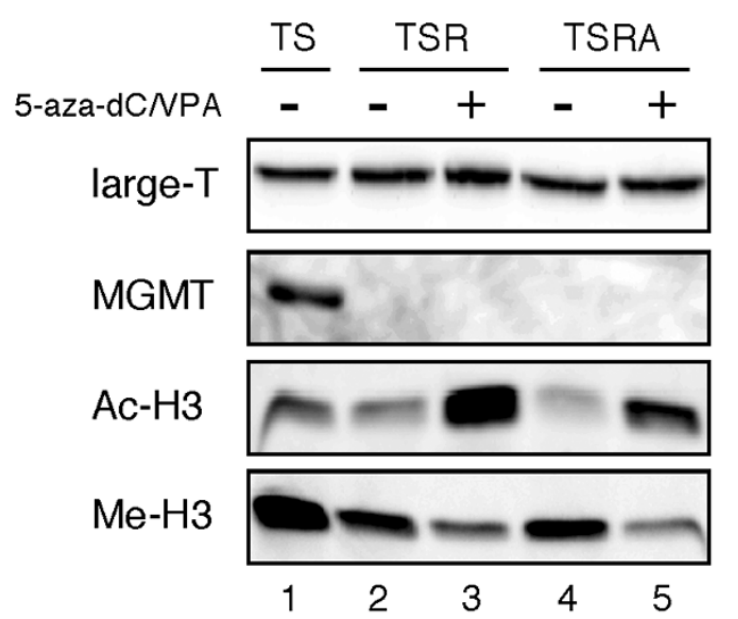

B

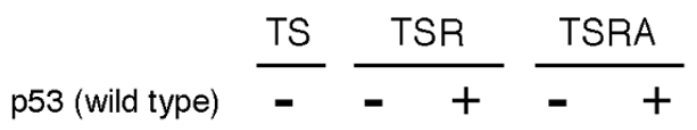

large- $\mathrm{T}$

p53

MGMT
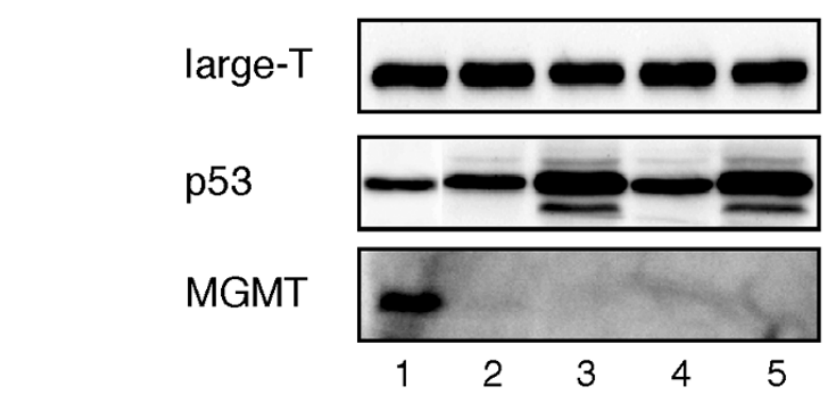

C

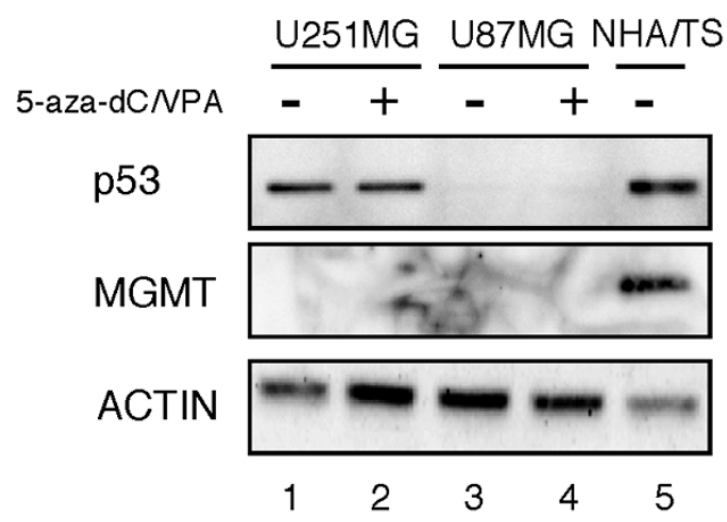

Figure 4

Neither treatment with 5-aza-dC/VPA nor p53 expression restores MGMT protein levels in transformed NHA cells. (A) Protein extracts $(10 \mu \mathrm{g})$ from the transformed NHA cells treated $(+)$ and untreated (-) with 5aza-dC/VPA were analyzed by immunoblotting. (B) Protein extracts $(10 \mu \mathrm{g})$ from the transformed NHA cells infected with wild type $\mathrm{p} 53(+)$ and control virus (-) were analyzed by immunoblotting. (C) Protein extracts ( $10 \mu \mathrm{g})$ from glioma cell lines treated $(+)$ and untreated $(-)$ with 5-aza-dC/VPA were analyzed by immunoblotting. pies for some glioma patients in combination with alkylating agents, such as temozolomide. Since VPA, which inhibits both class I and class II HDACs [29], has displayed potent in vitro and in vivo antitumor activities against brain tumor cells [30], it is one attractive candidate agent. The treatment with VPA appreciably inhibited growth of the NHA/TSRA cells in a dose dependent manner (Figure 6A) and two-week-treatment with $0.5 \mathrm{mM}$ VPA (within the range of serum levels achieved in pediatric high-grade glioma patients) [31] suppressed anchorage-independent growth (Figure 6B). Although hyperacetylation of histone $\mathrm{H} 3$ was noted in the NHA/ TSRA cells treated with VPA for two weeks, the treatment failed to restore protein (Figure 6C) and mRNA levels of MGMT (data not shown). Since protein levels of p21WAF1 and p27KIP1 were elevated in VPA-treated NHA/TSRA cells (Figure 6C), the antiproliferative effect might be mediated, at least in part, by cell cycle arrest, as widely acknowledged. These findings suggest that HDAC inhibitors, which suppress tumor cell growth without affecting MGMT expression, may be used in combination with alkylating agents to some glioma patients.

\section{Discussion}

Much of our understanding of the molecular basis of gliomagenesis derives from the study of established cell lines that are explanted from human tumors. Such cell lines are often assumed to be representative of the original diseases, and they have been extensively employed for the identification and preclinical testing of potential therapeutic compounds. However, they harbor an unknown number of genetic lesions, which complicates the study of specific molecules and pathways. Indeed, with respect to p53-mediated MGMT regulation, there have been contradictory observations, where transient knockdown of $p 53$ caused MGMT downregulation in SF767 glioma cells without affecting promoter methylation [28] but increased MGMT expression in T98 cells [27]. Since such discrepancy may depend on unidentified mutations specific for individual cell lines, it is difficult to provide constant conclusions using tumor-derived cell lines alone. Therefore, normal human cells as well as genetically engineered mouse models will prove very useful, which allow us to generate experimental models of human cancers by direct manipulation with defined genetic elements $[7,8]$.

Here we established glioma models from NHA cells and demonstrated that MGMT is downregulated in the transformed astrocyte cells. Although numerous studies have proposed the strong linkage between MGMT expression and promoter hypermethylation of the gene, treatment with epigenetic compounds (5-aza-dC/VPA) was unable to increase MGMT protein levels in the transformed NHA cells or in cultured tumor cells. Some previous reports demonstrated the epigenetic regulation of MGMT expres- 
Table 2: Diagnosis and p53 status of human gliomas: The index of Ki-67 staining and p53 status (mutation and immunopositivity) are listed with patient ID, age at surgery, gender (F, female; M, male), and clinical diagnosis (GBM, glioblastoma, WHO grade IV; AA, anaplastic astrocytoma, WHO grade III, AE anaplastic ependymoma, WHO grade III). Images of H\&E staining and immunohistochemistry are shown [see additional file I].

\begin{tabular}{|c|c|c|c|c|c|c|}
\hline ID & Age & Gender & Diagnosis & Ki-67 (\%) ${ }^{a}$ & p53 mutations $b$ & p53 staining $c$ \\
\hline GI & 73 & $\mathrm{~F}$ & GBM & 4.0 & $\mathrm{~N}$ & + \\
\hline G2 & 58 & $M$ & GBM & 44.3 & $\mathrm{R} 156 \mathrm{P}, \mathrm{H} 214 \mathrm{R}$ & ++ \\
\hline G3 & 36 & $M$ & GBM & 24.8 & $\mathrm{~N}$ & - \\
\hline G4 & 77 & $\mathrm{~F}$ & GBM & 92.5 & $\mathrm{~N}$ & - \\
\hline G5 & 53 & $M$ & GBM & 12.6 & $\mathrm{~N}$ & ++ \\
\hline G6 & 61 & $M$ & GBM & 43.6 & $\mathrm{~N}$ & + \\
\hline G7 & 83 & $M$ & GBM & 53.1 & HI93P & ++ \\
\hline G8 & 28 & $M$ & GBM & 29.0 & $\mathrm{H} 2 \mathrm{I} 4 \mathrm{R}$ & +++ \\
\hline G9 & 55 & $\mathrm{~F}$ & AA & 3.3 & $\mathrm{~N}$ & +++ \\
\hline GI0 & 42 & $\mathrm{~F}$ & $\mathrm{AE}$ & 42.6 & $\mathrm{~N}$ & - \\
\hline
\end{tabular}

aMIB-I labeling index (by anti-Ki-67 antibody) was analyzed using the MetaMorphV7.0 software (Molecular Cevices, Downingtown, PA, USA), by counting 500-2,000 nuclei per case.

${ }^{\mathrm{b}} \mathrm{p} 53 \mathrm{cDNA}$ was amplified by RT-PCR and the products were directly sequenced. $\mathrm{N}$, no mutation was detected.

cStaining intensity was classified into four categories: +++, strong; ++, moderate; +, weak; -, negative.

sion by treating tumor cell lines with 5 -aza-dC and testing the MGMT levels by RT-PCR $[32,33]$. However, based on the data presented here, such limited evaluation might not be sufficient, as RT-PCR assay overestimates the MGMT restoration. Since inappropriate evaluation would mislead the development of advanced therapies, protein levels or enzyme activity of MGMT in the cells treated with such compounds should be examined and compared with those in normal cells. In fact, the use of alkylating agents in combination with HDAC inhibitors has been hampered, probably because it has been suspected that such agents would increase MGMT expression. However, we showed that two-week-treatment with VPA inhibited tumor cell growth without increasing MGMT expression, suggesting potential clinical use although further preclinical studies are required.

As the treatment with 5-aza-dC/VPA slightly increased mRNA levels of MGMT in cultured glioma cells (including U87MG, U251MG and transformed NHA cells), methylation status might be involved in MGMT regulation to some extent. However, such treatment was insufficient to restore protein expression of MGMT, suggesting that the regulation mechanisms may largely depend on other cellular factors. Although p53 does not seem to contribute directly to MGMT expression, it is possible that other transcription factors, whose expressions have been downregulated or silenced during transformation and gliomagenesis, may collaborate with HDAC inhibitors to increase MGMT protein. Interestingly, the sonic hedgehog pathway has been shown to regulate the self-renewal of CD133-positive glioblastoma cells, which were resistant to temozolomide treatment [34]. Furthermore, some of CD133-positive glioma cultures highly expressed MGMT as well as target genes of the sonic hedgehog pathway
[35]. Thus, it is possible that the regulation of MGMT expression may be partially mediated by the sonic hedgehog pathway in some cases.

\section{Conclusion}

As discussed above, MGMT seems to be regulated by a number of ways in gliomas. The detailed mechanisms should be further analyzed as MGMT level is a critical determinant for efficacy of therapies with alkylating agents. Thus, identification of molecules and compounds that increase MGMT expression by screening NHA/TSR or NHA/TSRA cells with cDNA- and chemical-libraries would be very useful for the development of rational drug combination. We propose that the NHA cell system creates refined human glioma models for the systematic dissection of genetic alterations and elucidation of the complexities of the signaling pathways important for gliomagenesis. These systems also provide powerful means to find and authenticate molecules of particular promise for therapeutic targeting, and the present study provides an important proof-of-principle test for such systems.

\section{Methods \\ Clinical samples}

Brain tumor specimens were obtained, after informed consent, from patients undergoing tumor resection at the Kashiwaba Neurosurgical Hospital.

\section{Cell culture}

NHA cells (Cambrex Bio Science, Walkersville, MD, USA) were cultured in the astrocyte growth medium (AGM; Cambrex Bio Science). All other cells including immortalized NHA cells were maintained in Dulbecco's modified eagle medium (Seikagaku Co., Tokyo, Japan), supplemented with $10 \%$ fetal calf serum, $1 \mathrm{mM}$ Glutamine, 50 


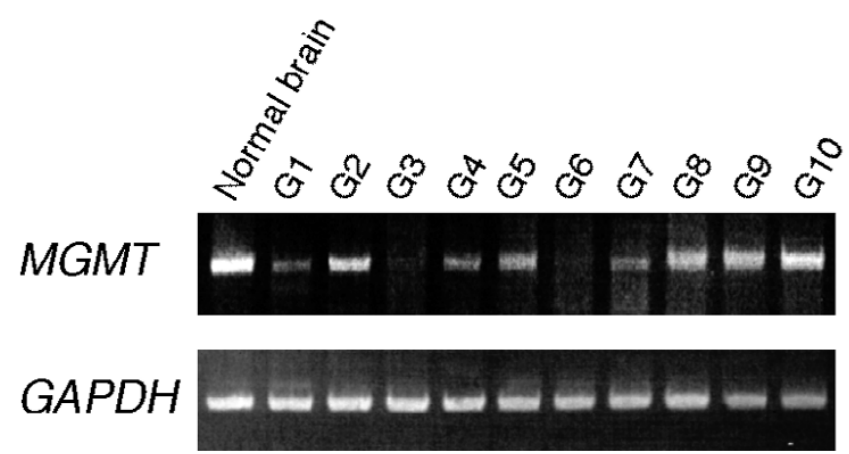

Figure 5

Expression of MGMT RNA in human glioma tissues.

The mRNA levels of MGMT expression in human glioma tissues were analyzed by semi-quantitative RT-PCR.

units/ml penicillin $\mathrm{G}$ and $50 \mu \mathrm{g} / \mathrm{ml}$ streptomycin. All cultures were incubated at $37^{\circ} \mathrm{C}$ under a humidified atmosphere of $95 \%$ air and 5\% CO2. For the combination treatment (5-aza-dC/VPA), 5-aza-dC (1 $\mu \mathrm{M}$; Sigma, St. Louis, MO, USA) was added for an initial incubation of 48 $\mathrm{h}$, after which VPA ( $1 \mathrm{mM}$; Sigma) was added for an additional $24 \mathrm{~h}$.

\section{Retroviral vectors and retroviral-mediated gene transfer}

A cDNA fragment encoding murine ecotropic retrovirus receptor $(E c o V R)$ was obtained from the retroviral plasmid pCX4hyg-EcoVR [14], and then subcloned into pCX4redEx vector [GenBank: AB296084]. Myc-His-tagged active form of mouse AKT1 cDNA, which has N-terminal myristoylation, was isolated from the pUSEamp-myr-AKT plasmid (Upstate, Charlottesville, VA, USA) and subcloned into pCX4bleo retroviral vector [GenBank: AB086388]. Full-length cDNAs for human MGMT and p53 were generated by PCR and subcloned into pCX4bleo and pCX4gfp [GenBank: AB296083] retroviral vectors, respectively. Primer sequences used in this experiment included 5'-ATG GAC AAG GAT TGT GAA-3' and 5'-TCA GTT TCG GCC AGC AGG-3' for human MGMT and 5'CTG AAT TCA TGG AGG AGC CGC AGT CAG-3' and 5'CCG AAT TCA GTC TGA GTC AGG CCC TTC-3' for human p53. Other retroviral vectors and the procedure of retroviral-mediated gene transfer were described previously [14]. The murine EcoVR was first introduced into NHA cells by using amphotropic virus, in order to make human cells susceptible to the subsequent infection with ecotropic viral vectors. Infected cell populations were selected in blasticidin S $(20 \mu \mathrm{g} / \mathrm{ml}), \mathrm{G} 418(1000 \mu \mathrm{g} / \mathrm{ml})$, puromycin (500 ng/ml), or zeocine $(500 \mu \mathrm{g} / \mathrm{ml})$ for two weeks. In all cases, cultures arose from polyclonal expansion of infected cells.

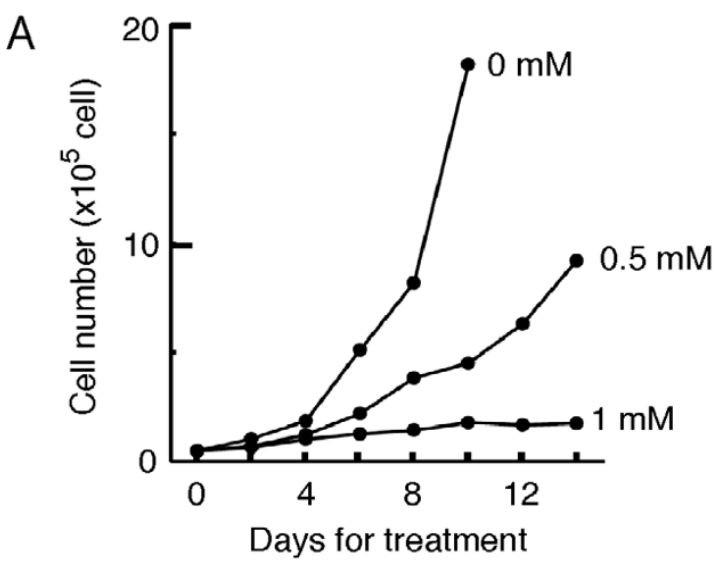

B
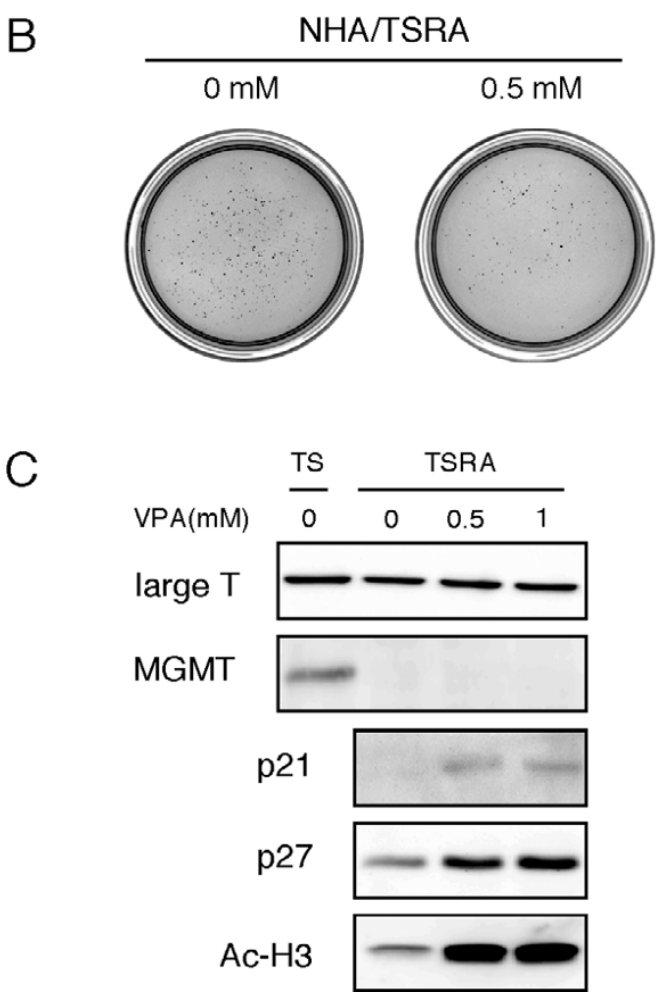

Figure 6

VPA inhibits cell growth in transformed human astrocyte cells. (A) Dose-dependent anti-proliferative effects of VPA were shown. NHA/TSRA cells were treated with 0, 0.5 and I.0 mM VPA for I 4 days. Cell numbers were counted every other day and results were expressed in the mean of two independent experiments. (B) NHA/TSRA cells, treated $(0.5 \mathrm{mM})$ and untreated $(0 \mathrm{mM})$ with VPA for two weeks, were subjected to soft-agar colony formation assay. 2 $\times 10^{4}$ cells were plated, incubated at $37^{\circ} \mathrm{C}$ for 21 days and stained with 3-(4,5-dimethyl-thiazol-2-yl)-2,5-diphenyltetrazolium bromide. Note VPA was not included in the assay media. (C) Protein extracts ( $10 \mu \mathrm{g})$ from NHA/TSRA cells treated with VPA were analyzed by immunoblotting using indicated antibodies. 


\section{RT-PCR}

Total RNA was isolated with the TRI Reagent (Sigma) and reverse transcribed into cDNA using the oligo-dT primer (Invitrogen, Carlsbad, CA, USA) and the Superscript II (Invitrogen). The levels of MGMT were analyzed by PCR with the KOD plus DNA polymerase (Toyobo, Tokyo, Japan) using the primers described above. PCR primers for Glyceraldehydes-3-phosphate dehydrogenase (GAPDH) were described previously [15].

\section{Soft-agar colony formation assay and xenograft propagation}

Soft-agar colony formation assay [15] and xenograft propagation [36] were carried out as described. Female athymic nude mice (BALB/CAJcl-nu/nu) were purchased from Clea Japan (Tokyo, Japan) and all animal procedures were carried out according to the protocol approved by the institutional Animal Care and Use Committee at Hokkaido University Graduate School of Medicine.

\section{Histological analysis and immunohistochemistry}

Formalin-fixed paraffin-embedded tissues were sectioned and stained with haematoxylin and eosin (H\&E) using standard protocols. Immunohistochemistry was performed using anti-Ki-67 (MIB-1; Dako, Glostrup, Denmark) and anti-p53 (DO-7; Dako) monoclonal antibodies.

\section{Immunoblotting}

Protein determination, SDS-PAGE and immunoblotting were carried out as described previously [37], and reactive protein signals were visualized by chemiluminescence using the ECL reagent (Amersham, Piscataway, NJ, USA) or the SuperSignal West Femto reagent (Pierce, Rockford, IL, USA). Antibodies were obtained from the following sources: anti-SV40 large T antigen (Ab-1) and anti-SV 40 small $\mathrm{t}$ antigen (Ab-3) monoclonal antibodies (Oncogene Research Product, San Diego, CA, USA); anti-p53 and anti-AKT polyclonal antibodies (Cell Signaling Technology, Beverly, MA, USA); anti-RAS and anti-p27KIP1 monoclonal antibodies (Transduction Laboratories, Lexington, KY, USA); anti-dimethylated Histone-H3 (Me-H3) and anti-acetylated Histone-H3 (Ac-H3) polyclonal antibodies (Upstate); anti-MGMT (MT3.1) and anti-ACTIN monoclonal antibodies (Chemicon International, Temecula, CA, USA); an anti-p21 ${ }^{\text {WAF1 }}$ monoclonal antibody (Ab-1; Calbiochem, San Diego, CA, USA); an anti-hTERT (L20) polyclonal antibody (Santa Cruz Biotechnology, Santa Cruz, CA, USA).
Abbreviations
MGMT: $\mathrm{O}^{6}$-methylguanine-DNA methyltransferase
GBM: glioblastoma multiforme

NHA: normal human astrocyte

hTERT (T): human telomerase catalytic subunit

H-RasV12 (R): activated H-Ras

myrAKT (A): myristoylated form (active form) of AKT

SV40ER (S): simian virus 40 early region

HDAC: histone deacetylase

VPA: Valproic acid

5-aza-dC: 5-aza-2'-deoxycytosine

RT-PCR: reverse transcriptase polymerase chain reaction

GAPDH: Glyceraldehydes-3-phosphate dehydrogenase

\section{Competing interests}

The author(s) declare that they have no competing interests.

\section{Authors' contributions}

KS designed the research, carried out all experiments except for immunohistochemistry, and drafted the manuscript. TA participated in the design of the study and contributed to new reagents. EA carried out H\&E staining and immunohistochemistry. KT participated in epigenetic studies. SK collected and analyzed clinical samples. ST carried out histological analyses, conceived of the study, participated in its design and coordination, and helped to draft the manuscript. All authors read and approved the final manuscript.

\section{Additional material}

\section{Additional file 1}

Histopathological analysis of human brain tumors. Formalin-fixed paraffin-embedded tissue sections were stained with H\&E. Low- $(\times 100)$ and high- (×400) magnification images are shown (bar in G1, $200 \mu \mathrm{m})$. Tissue sections were also processed for immunohistochemistry using Ki-67 and p53 antibodies. Staining intensities were summarized in Table 2. Click here for file

[http://www.biomedcentral.com/content/supplementary/14764598-6-36-S1.pdf]

\section{Acknowledgements}

We thank Jared Ordway (Orion Genomics, St. Louis, MO), Christopher Calabrese (St. Jude Children's Research Hospital, Memphis, TN) and Taiko Sukezane (KAN Research Institute, Kobe, Japan) for their helpful discussion and critical reading of this manuscript. We thank Tomoyuki Shishido (Nara Institute of Science and Technology, Ikoma, Japan) for providing the reagents and Miho Nodagashira (Hokkaido University, Sapporo, Japan) for her 
excellent technical assistance. This work was supported by the Mochida Memorial Foundation for Medical and Pharmaceutical Research (to ST), the Suhara Memorial Foundation (to ST), and Grant-in-Aid from Ministry of Education, Culture, Sports, Science and Technology of Japan (to ST).

\section{References}

I. Kleihues P, Burger PC, Scheithauer BW: The new WHO classification of brain tumours. Brain Pathol 1993, 3:255-268.

2. Kleihues P, Louis DN, Scheithauer BW, Rorke LB, Reifenberger G, Burger PC, Cavenee WK: The WHO classification of tumors of the nervous system. J Neuropathol Exp Neurol 2002, 6 I:2 I5-225. discussion 226-219

3. Ohgaki H, Dessen P, Jourde B, Horstmann S, Nishikawa T, Di Patre PL, Burkhard C, Schuler D, Probst-Hensch NM, Maiorka PC, et al.: Genetic pathways to glioblastoma: a population-based study. Cancer Res 2004, 64:6892-6899.

4. Reardon DA, Rich JN, Friedman HS, Bigner DD: Recent advances in the treatment of malignant astrocytoma. J Clin Oncol 2006, 24: $\mid 253-1265$

5. Stupp R, Gander M, Leyvraz S, Newlands E: Current and future developments in the use of temozolomide for the treatment of brain tumours. Lancet Oncol 2001, 2:552-560.

6. Gerson SL: MGMT: its role in cancer aetiology and cancer therapeutics. Nat Rev Cancer 2004, 4:296-307.

7. Akagi T: Oncogenic transformation of human cells: shortcomings of rodent model systems. Trends Mol Med 2004, I 0:542-548.

8. Zhao JJ, Roberts TM, Hahn WC: Functional genetics and experimental models of human cancer. Trends Mol Med 2004, 10:344-350.

9. Hu X, Holland EC: Applications of mouse glioma models in preclinical trials. Mutat Res 2005, 576:54-65.

10. Sonoda Y, Ozawa T, Hirose Y, Aldape KD, McMahon M, Berger MS Pieper RO: Formation of intracranial tumors by genetically modified human astrocytes defines four pathways critical in the development of human anaplastic astrocytoma. Cancer Res 2001, 61:4956-4960.

II. Sonoda Y, Ozawa T, Aldape KD, Deen DF, Berger MS, Pieper RO: Akt pathway activation converts anaplastic astrocytoma to glioblastoma multiforme in a human astrocyte model of glioma. Cancer Res 2001, 6 1:6674-6678.

12. Colella S, Ohgaki H, Ruediger R, Yang F, Nakamura M, Fujisawa H, Kleihues P, Walter G: Reduced expression of the Aalpha subunit of protein phosphatase $2 A$ in human gliomas in the absence of mutations in the Aalpha and Abeta subunit genes. Int I Cancer 200I, 93:798-804.

13. Arroyo JD, Hahn WC: Involvement of PP2A in viral and cellular transformation. Oncogene 2005, 24:7746-7755.

14. Akagi T, Sasai K, Hanafusa $\mathrm{H}$ : Refractory nature of normal human diploid fibroblasts with respect to oncogene-mediated transformation. Proc Natl Acad Sci USA 2003, 100: | 3567-13572.

15. Sasai K, Kakumoto K, Hanafusa H, Akagi T: The Ras-MAPK pathway downregulates Caveolin- I in rodent fibroblast but not in human fibroblasts: implications in the resistance to oncogene-mediated transformation. Oncogene 2007, 26:449-455.

16. Kakumoto K, Sasai K, Sukezane T, Oneyama C, Ishimaru S, Shibutani K, Mizushima $H$, Mekada E, Hanafusa H, Akagi T: FRA I is a determinant for the difference in RAS-induced transformation between human and rat fibroblasts. Proc Natl Acad Sci USA 2006, I 03:5490-5495.

17. Sukezane T, Oneyama C, Kakumoto K, Shibutani K, Hanafusa H, Akagi T: Human diploid fibroblasts are resistant to MEK/ERKmediated disruption of the actin cytoskeleton and invasiveness stimulated by Ras. Oncogene 2005, 24:5648-5655.

18. Silber JR, Mueller BA, Ewers TG, Berger MS: Comparison of O6methylguanine-DNA methyltransferase activity in brain tumors and adjacent normal brain. Cancer Res 1993, 53:3416-3420.

19. Ordway JM, Fenster SD, Ruan H, Curran T: A transcriptome map of cellular transformation by the fos oncogene. Mol Cancer 2005, 4:19.

20. A transcriptome map of cellular transformation by the Fos oncogene [http://www.stjuderesearch.org/v-fosSOM/]

21. Zhu WG, Otterson GA: The interaction of histone deacetylase inhibitors and DNA methyltransferase inhibitors in the treatment of human cancer cells. Curr Med Chem Anticancer Agents 2003, 3:187-199.

22. Russell SJ, Ye YW, Waber PG, Shuford M, Schold SC Jr, Nisen PD: p53 mutations, O6-alkylguanine DNA alkyltransferase activity, and sensitivity to procarbazine in human brain tumors. Cancer 1995, 75:1339-1342.

23. Rolhion C, Penault-Llorca F, Kemeny JL, Kwiatkowski F, Lemaire J, Chollet P, Finat-Duclos F, Verrelle P: O(6)-methylguanine-DNA methyltransferase gene (MGMT) expression in human glioblastomas in relation to patient characteristics and p53 accumulation. Int J Cancer 1999, 84:416-420.

24. Yuan $\mathrm{Q}$, Matsumoto $\mathrm{K}$, Nakabeppu $\mathrm{Y}$, Iwaki T: A comparative immunohistochemistry of O6-methylguanine-DNA methyltransferase and p53 in diffusely infiltrating astrocytomas. Neuropathology 2003, 23:203-209.

25. Roos WP, Batista LF, Naumann SC, Wick W, Weller M, Menck CF, Kaina $B$ : Apoptosis in malignant glioma cells triggered by the temozolomide-induced DNA lesion O6-methylguanine. Oncogene 2007, 26:186-197.

26. Ishii N, Maier D, Merlo A, Tada M, Sawamura Y, Diserens AC, Van Meir EG: Frequent co-alterations of TP53, pI6/CDKN2A, pI4ARF, PTEN tumor suppressor genes in human glioma cell lines. Brain Pathol 1999, 9:469-479.

27. Natsume A, Ishii D, Wakabayashi T, Tsuno T, Hatano H, Mizuno M, Yoshida J: IFN-beta down-regulates the expression of DNA repair gene MGMT and sensitizes resistant glioma cells to temozolomide. Cancer Res 2005, 65:7573-7579.

28. Blough MD, Zlatescu MC, Cairncross JG: O6-methylguanineDNA methyltransferase regulation by $\mathrm{p} 53$ in astrocytic cells. Cancer Res 2007, 67:580-584.

29. Gottlicher M, Minucci S, Zhu P, Kramer OH, Schimpf A, Giavara S Sleeman JP, Lo Coco F, Nervi C, Pelicci PG, et al.: Valproic acid defines a novel class of HDAC inhibitors inducing differentiation of transformed cells. Embo / 200I, 20:6969-6978.

30. Bacon CL, Gallagher HC, Haughey JC, Regan CM: Antiproliferative action of valproate is associated with aberrant expression and nuclear translocation of cyclin D3 during the C6 glioma GI phase. J Neurochem 2002, 83:12-19.

31. Wagner S, Csatary CM, Gosztonyi G, Koch HC, Hartmann C, Peters O, Hernaiz-Driever P, Theallier-Janko A, Zintl F, Langler A, et al: Combined treatment of pediatric high-grade glioma with the oncolytic viral strain $\mathrm{MTH}-68 / \mathrm{H}$ and oral valproic acid. Apmis 2006, I | 4:73 I-743.

32. Hoon DS, Spugnardi M, Kuo C, Huang SK, Morton DL, Taback B: Profiling epigenetic inactivation of tumor suppressor genes in tumors and plasma from cutaneous melanoma patients. Oncogene 2004, 23:4014-4022.

33. Danam RP, Howell SR, Brent TP, Harris LC: Epigenetic regulation of O6-methylguanine-DNA methyltransferase gene expression by histone acetylation and methyl-CpG binding proteins. Mol Cancer Ther 2005, 4:6I-69.

34. Clement V, Sanchez P, de Tribolet N, Radovanovic I, Ruiz i Altaba A: HEDGEHOG-GLII signaling regulates human glioma growth, cancer stem cell self-renewal, and tumorigenicity. Curr Biol 2007, I 7: 165-I72.

35. Liu G, Yuan X, Zeng Z, Tunici P, Ng H, Abdulkadir IR, Lu L, Irvin D, Black KL, Yu JS: Analysis of gene expression and chemoresistance of CDI33+ cancer stem cells in glioblastoma. Mol Cancer 2006, 5:67.

36. Sasai K, Romer JT, Lee Y, Finkelstein D, Fuller C, McKinnon PJ, Curran T: Shh pathway activity is down-regulated in cultured medulloblastoma cells: implications for preclinical studies. Cancer Res 2006, 66:4215-4222.

37. Akagi T, Murata $\mathrm{K}$, Shishido T, Hanafusa $\mathrm{H}$ : v-Crk activates the phosphoinositide 3-kinase/AKT pathway by utilizing focal adhesion kinase and H-Ras. Mol Cell Biol 2002, 22:7015-7023. 